\title{
Investigation of Switching-Time Variations for Nanoscale MOSFETs Using the Effective-Drive-Current Approach
}

\author{
Yu-Sheng Wu, Student Member, IEEE, Ming-Long Fan, Student Member, IEEE, and Pin Su, Member, IEEE
}

\begin{abstract}
This letter investigates the impacts of random dopant fluctuation (RDF) and line edge roughness (LER) on the switching-time (ST) variation for nanoscale MOSFETs using the effective-drive-current $\left(I_{\text {eff }}\right)$ approach that decouples the ST variation into transition-charge $(\Delta Q)$ and $I_{\text {eff }}$ variations. Although the RDF has been recognized as the main variation source to the threshold-voltage variation, this letter indicates that the relative importance of LER increases as the ST variation is considered.
\end{abstract}

Index Terms-Line edge roughness (LER), MOSFET, random dopant fluctuation (RDF), switching time (ST).

\section{INTRODUCTION}

W ITH MOSFET scaling, the impact of random dopant fluctuation (RDF) and line edge roughness (LER) on the threshold-voltage $\left(V_{\mathrm{th}}\right)$ variation of nanoscale transistors is growing and being extensively examined [1]-[3]. For example, Asenov et al. [2] has shown that, for a given LER variation, as gate dimensions are reduced, the $V_{\text {th }}$ fluctuations increase and are comparable in magnitude to those caused by RDF. Roy et al. [3] has concluded that the $V_{\text {th }}$ variation due to RDF would dominate the behavior of the bulk MOSFETs if the LER can meet the prescription of the International Technology Roadmap for Semiconductors.

For logic circuits, however, the variation of signal switching time (ST) due to RDF and LER is particularly important. Whether there is any gap between $V_{\mathrm{th}}$ and ST variations merits investigation. In this letter, we investigate the ST variation due to RDF and LER for bulk MOSFETs using the approach of effective drive current in CMOS inverters [4].

\section{Methodology}

We decouple the ST variation into transition-charge $(\Delta Q)$ and effective-drive-current $\left(I_{\text {eff }}\right)$ variations. The ST can be defined as $\Delta Q / I_{\text {eff }}$ [5], where $\Delta Q$ is the transition

Manuscript received June 2, 2009; revised October 30, 2009. First published December 22, 2009; current version published January 27, 2010. This work was supported in part by the National Science Council of Taiwan under Contract NSC 98-2221-E-009-178 and in part by the Ministry of Education in Taiwan under the ATU Program. The review of this letter was arranged by Editor K. De Meyer.

The authors are with the Department of Electronics Engineering and Institution of Electronics, National Chiao Tung University, Hsinchu 30013, Taiwan (e-mail: pinsu@falculty.nctu.edu.tw).

Color versions of one or more of the figures in this letter are available online at http://ieeexplore.ieee.org.

Digital Object Identifier 10.1109/LED.2009.2037247 charge between logic "ON" and "OFF" states. The $\Delta Q$ for an NFET can be calculated by $Q_{n}\left(V_{\mathrm{GS}}=V_{\mathrm{DD}}, V_{\mathrm{DS}}=\right.$ $0 \mathrm{~V})-Q_{n}\left(V_{\mathrm{GS}}=0 \mathrm{~V}, V_{\mathrm{DS}}=V_{\mathrm{DD}}\right)$. The $I_{\mathrm{eff}}$ for an NFET can be approximated as $\left[I_{\mathrm{DS}}\left(V_{\mathrm{GS}}=V_{\mathrm{DD}}, V_{\mathrm{DS}}=0.5 V_{\mathrm{DD}}\right)+\right.$ $\left.I_{\mathrm{DS}}\left(V_{\mathrm{GS}}=0.5 V_{\mathrm{DD}}, V_{\mathrm{DS}}=V_{\mathrm{DD}}\right)\right] / 2[4]$. Therefore, in contrast to the time-consuming mixed-mode transient simulation, only dc simulation for a single device is needed to derive $\Delta Q$ and $I_{\text {eff }}$. More importantly, the effective-drive-current approach may provide physical insights in the assessment of ST variations.

The device parameters of bulk MOSFETs used in this letter are gate length $\left(L_{\text {gate }}\right)=25 \mathrm{~nm}$, channel width $(\mathrm{W})=25 \mathrm{~nm}$, oxide thickness $\left(t_{\mathrm{ox}}\right)=0.8 \mathrm{~nm}$, source/drain junction depth $\left(x_{j}\right)=12.5 \mathrm{~nm}$, channel doping $\left(N_{\mathrm{ch}}\right)=4.8 \times 10^{18} \mathrm{~cm}^{-3}$, and supply voltage $\left(V_{\mathrm{DD}}\right)=0.8 \mathrm{~V}$. To assess the RDF in bulk MOSFETs, we have carried out the atomistic device simulation using the Monte Carlo approach to generate the dopants in the channel [1]. To avoid the charge trapping in the sharp Coulomb potential well and, hence, the mesh size dependences of the simulation results, we have employed the density gradient method in our atomistic simulation [3]. The boundary condition at the $\mathrm{Si} / \mathrm{SiO}_{2}$ interface for the density gradient method is that the carrier density changes continuously across the interface, i.e., the continuity of the wavefunctions across the interface [6]. Fig. 1(a) shows one of the 150 samples in our atomistic simulation. To assess the LER, the line edge patterns were derived using the Fourier synthesis approach similar to the one in [2], and then, the Monte Carlo simulation was performed. The parameters used in the LER simulation are the rms amplitude $\Delta=1 \mathrm{~nm}$ [7] and the correlation length $\Lambda=30 \mathrm{~nm}$. Fig. 1(b) shows one of the 150 samples in our simulation. In this letter, we use the drift-diffusion equation as the transport model. The velocity saturation model is used to assess the on-current under the high drain field.

\section{RESUlTS AND DisCUSSION}

Fig. 2(a) compares the saturation threshold-voltage $\left(V_{\text {th,sat }}\right)$ distributions due to RDF and LER for bulk MOSFETs. Fig. 2(b) compares the ST distributions due to the RDF and LER. It can be seen that the standard deviation of $V_{\mathrm{th} \text {,sat }}\left(\sigma V_{\mathrm{th} \text {,sat }}\right)$ due to RDF is larger than that due to LER. Nevertheless, Fig. 2(b) shows that the standard deviation of ST $(\sigma \mathrm{ST})$ due to LER is comparable with that due to RDF. In other words, the relative importance of LER for ST variation increases as compared with 


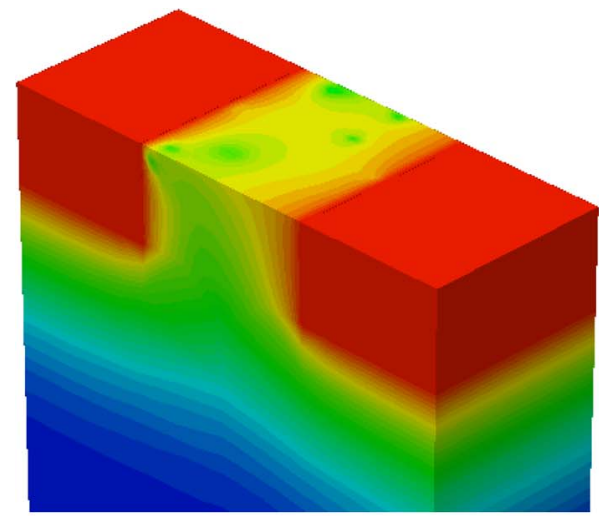

(a)

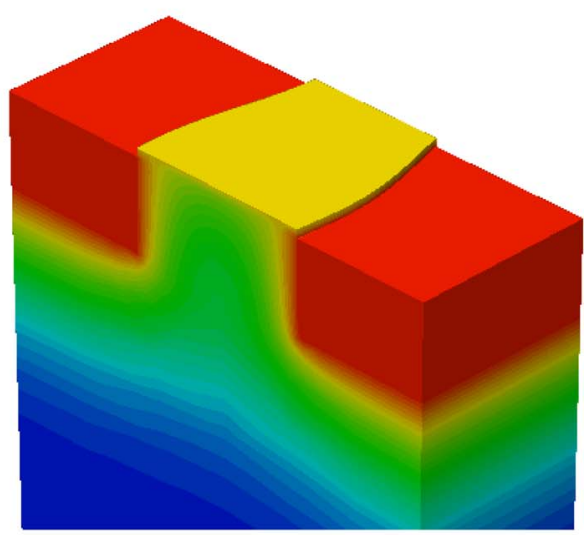

(b)

Fig. 1. Simulated bulk MOSFETs in this letter. (a) One of the samples with RDF and (b) one of the samples with LER.
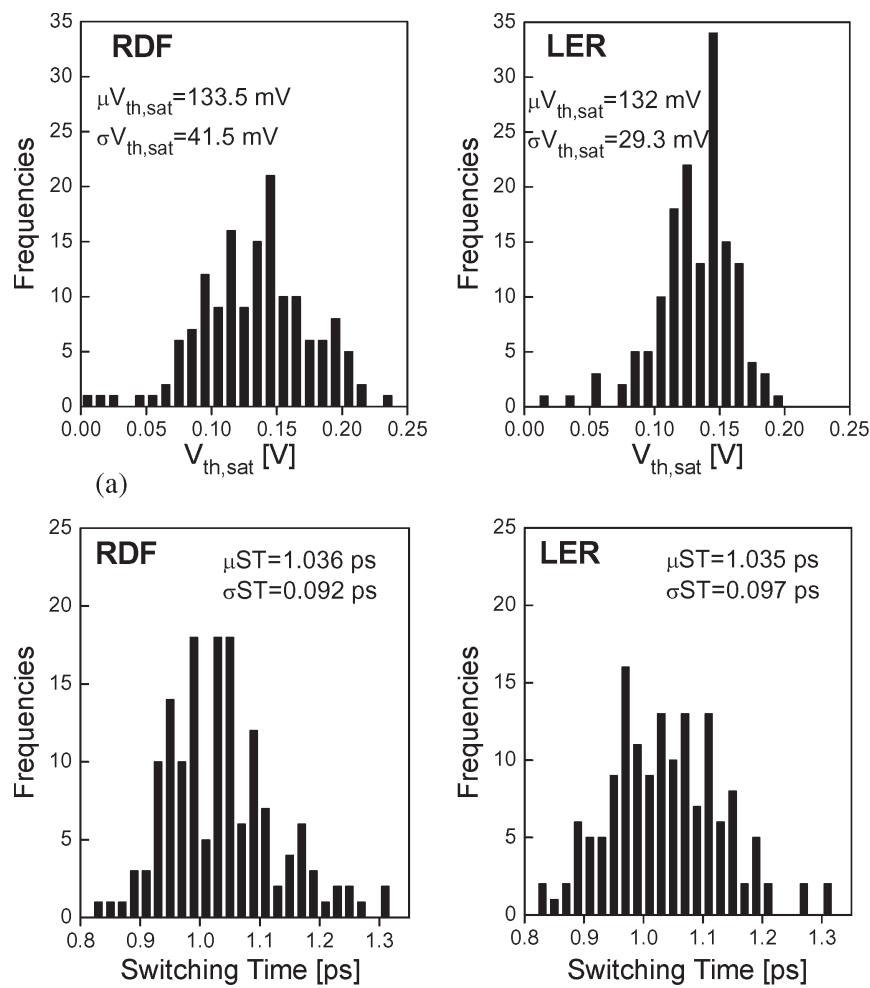

(b)

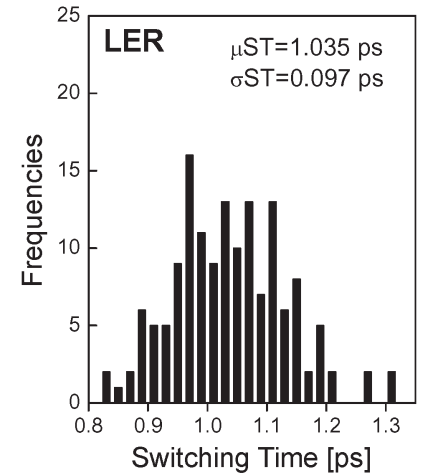

Fig. 2. (a) $V_{\text {th,sat }}$ distribution of 150 samples due to RDF and LER. (b) ST distribution of 150 samples due to RDF and LER.

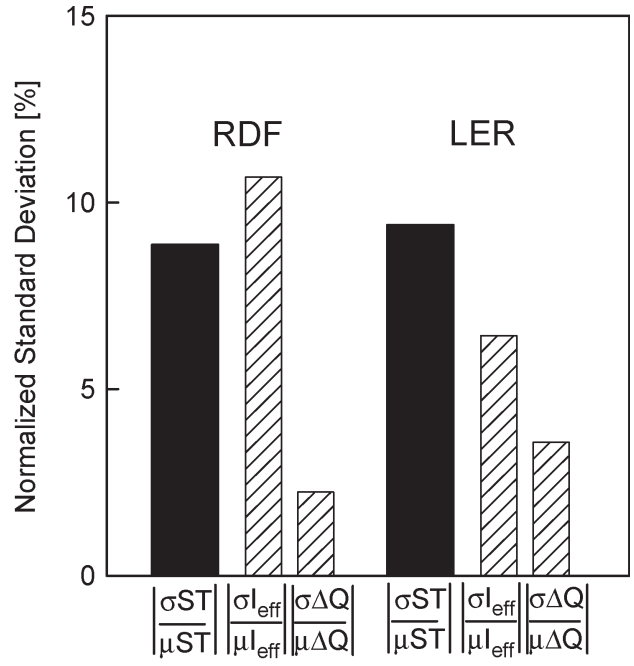

Fig. 3. Normalized standard deviations of ST, $I_{\text {eff }}$, and $\Delta Q$ due to RDF and LER.

that for $V_{\mathrm{th}, \text { sat }}$ variation. Since $\mathrm{ST}=\Delta Q / I_{\mathrm{eff}}$, the normalized standard deviation of ST $(\sigma \mathrm{ST} / \mu \mathrm{ST})$ can be approximated as $|\sigma \mathrm{ST} / \mu \mathrm{ST}| \approx\left|\sigma \Delta Q / \mu \Delta Q-\sigma I_{\text {eff }} / \mu I_{\text {eff }}\right|$, where $\mu \mathrm{ST}, \mu \Delta Q$, and $\mu I_{\text {eff }}$ are the mean values of ST, $\Delta Q$, and $I_{\text {eff }}$, respectively. In this letter, we consider the standard deviations $\sigma \mathrm{ST}, \sigma \Delta Q$, and $\sigma I_{\text {eff }}$ as signed numbers. Fig. 3 shows the $|\sigma \mathrm{ST} / \mu \mathrm{ST}|$, $|\sigma \Delta Q / \mu \Delta Q|$, and $\left|\sigma I_{\text {eff }} / \mu I_{\text {eff }}\right|$ (normalized standard deviation of ST, $\Delta Q$, and $I_{\text {eff }}$, respectively) caused by RDF and LER. It can be seen that the $|\sigma \mathrm{ST} / \mu \mathrm{ST}|$ due to RDF is roughly equal to the difference of $\left|\sigma I_{\text {eff }} / \mu I_{\text {eff }}\right|$ and $|\sigma \Delta Q / \mu \Delta Q|$ due to RDF. However, the $|\sigma \mathrm{ST} / \mu \mathrm{ST}|$ due to LER is roughly equal to the sum of $\left|\sigma I_{\mathrm{eff}} / \mu I_{\mathrm{eff}}\right|$ and $|\sigma \Delta Q / \mu \Delta Q|$ due to LER. The results in Fig. 3 can be explained as follows. The impact of RDF on MOSFETs stems from the variation of the effective channel doping $\left(N_{\text {ch,eff }}\right)$. For devices with smaller $N_{\text {ch,eff }}$ values, the $V_{\text {th }}$ is smaller, and hence, $I_{\text {eff }}$ and $\Delta Q$ are larger because they are roughly proportional to $\left(V_{\mathrm{GS}}-V_{\mathrm{th}}\right)$. Thus, $I_{\text {eff }}$ and $\Delta Q$ are positively correlated [Fig. 4(a)]. Therefore, $|\sigma \mathrm{ST} / \mu \mathrm{ST}|$ is roughly equal to the difference between $|\sigma \Delta Q / \mu \Delta Q|$ and $\left|\sigma I_{\mathrm{eff}} / \mu I_{\mathrm{eff}}\right|$ because the quantities of $\sigma \Delta Q$ and $\sigma I_{\text {eff }}$ have the same sign. In other words, the impacts of $\mathrm{RDF}$ on $\Delta Q$ and $I_{\text {eff }}$ are mutually canceled, and $|\sigma \mathrm{ST} / \mu \mathrm{ST}|$ is reduced.

The impact of LER on MOSFETs results from the variation of the effective channel length $\left(L_{\text {eff }}\right)$. For devices with shorter $L_{\text {eff' }}$ 's, the $V_{\text {th }}$ is smaller because of the short-channel effect, and hence, the $I_{\text {eff }}$ is larger. As for $\Delta Q$, devices with shorter $L_{\text {eff's }}$ 'possess smaller $\Delta Q$ 's because $\Delta Q$ is proportional to the gate area $\left(W \times L_{\text {eff }}\right)$. Thus, $I_{\text {eff }}$ and $\Delta Q$ are negatively correlated [Fig. 4(b)]. Therefore, $|\sigma \mathrm{ST} / \mu \mathrm{ST}|$ is roughly equal to the sum of $|\sigma \Delta Q / \mu \Delta Q|$ and $\left|\sigma I_{\text {eff }} / \mu I_{\text {eff }}\right|$ because the quantities of $\sigma \Delta Q$ and $\sigma I_{\text {eff }}$ have the opposite sign. In other words, the $|\sigma \mathrm{ST} / \mu \mathrm{ST}|$ is larger than either $|\sigma \Delta Q / \mu \Delta Q|$ or $\left|\sigma I_{\text {eff }} / \mu I_{\text {eff }}\right|$.

It should be noted that, for ultrascaled devices in which ballistic transport becomes significant, the $I_{\text {eff }}$ will be underestimated if the drift-diffusion model is used. However, the normalized standard deviation of $I_{\text {eff }}\left(\sigma I_{\text {eff }} / \mu I_{\text {eff }}\right)$ may not be 

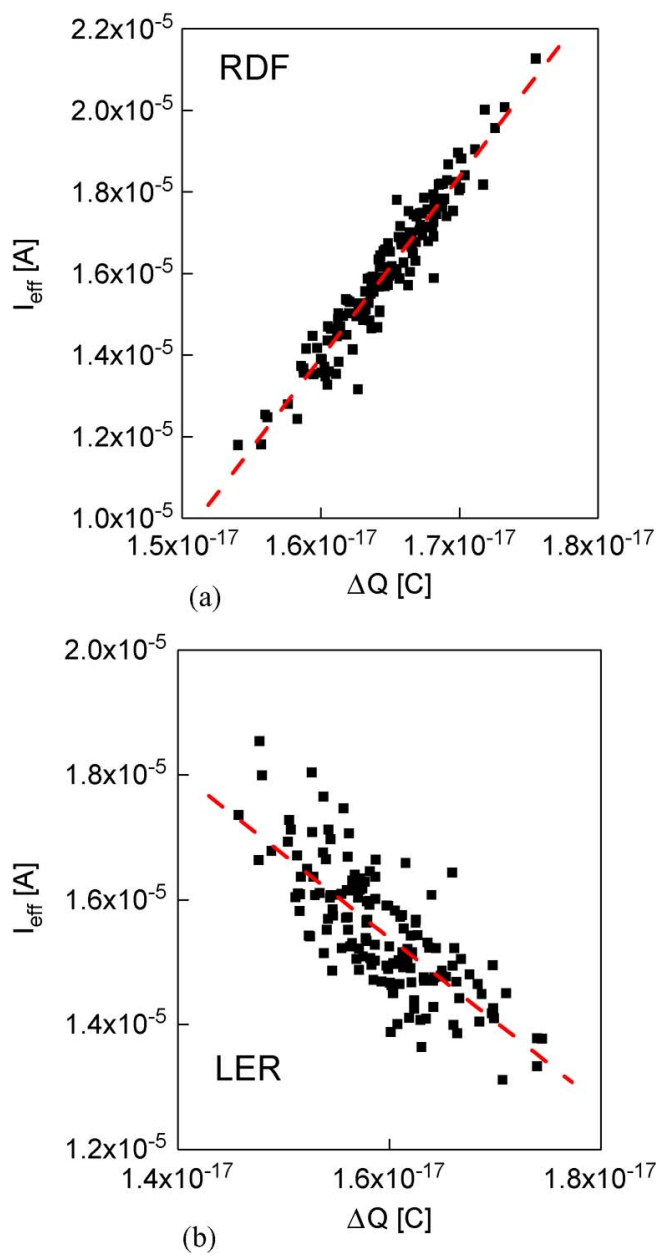

Fig. 4. Correlations of $I_{\text {eff }}$ and $\Delta Q$ distributions for MOSFETs with (a) RDF and (b) LER.

as sensitive to the transport model as the $I_{\text {eff }}$. Aside from the electrostatic potential fluctuation, the discrete dopants will also cause the mobility fluctuation, which is not considered in this letter. Therefore, the $\sigma I_{\text {eff }}$ due to RDF may be larger than that in Fig. 3 because of the mobility fluctuation caused by discrete dopants.

\section{CONCLUSION}

We have investigated the impacts of RDF and LER on the ST variation for nanoscale MOSFETs using the effective-drivecurrent approach that decouples the $\mathrm{ST}$ variation into $\Delta Q$ and $I_{\text {eff variations. Our results indicate that the ST variation caused }}$ by LER may be larger than that caused by RDF. This is because $I_{\text {eff }}$ and $\Delta Q$ variations due to RDF are mutually canceled and the ST variation caused by RDF is reduced, while $I_{\mathrm{eff}}$ and $\Delta Q$ variations due to LER increase the ST variation caused by LER. Although the RDF has been recognized as the main variation source to $V_{\mathrm{th}}$ variation, the relative importance of LER increases as the ST variation is considered. This letter may provide insights for device and circuit designs using advanced CMOS technology.

\section{REFERENCES}

[1] D. J. Frank, Y. Taur, M. Ieong, and H. P. Wong, "Monte Carlo modeling of threshold variation due to dopant fluctuations," in VLSI Symp. Tech. Dig., 1999 , p. 169.

[2] A. Asenov, S. Kaya, and A. R. Brown, "Intrinsic parameter fluctuation in decananometer MOSFETs introduced by gate line edge roughness," IEEE Trans. Electron Devices, vol. 50, no. 5, pp. 1254-1260, May 2003.

[3] G. Roy, A. R. Brown, F. A. Lema, S. Roy, and A. Asenov, "Simulation study of individual and combined sources of intrinsic parameter fluctuations in conventional nano-MOSFETs," IEEE Trans. Electron Devices, vol. 53, no. 12, pp. 3063-3070, Dec. 2006.

[4] M. H. Na, E. J. Nowak, W. Haensch, and J. Cai, "The effective drive current in CMOS inverters," in IEDM Tech. Dig., 2002, pp. 121-124.

[5] S. E. Laux, "A simulation study of the switching times of 22- and 17-nm gate-length SOI nFETs on high mobility substrates and Si," IEEE Trans. Electron Devices, vol. 54, no. 9, pp. 2304-2320, Sep. 2007.

[6] A. Wettstein, O. Penzin, and E. Lyumkis, "Integration of the density gradient model into a general purpose device simulator," VLSI Design, vol. 15, no. 4, pp. 751-759, Jan. 2002.

[7] International Technology Roadmap for Semiconductors. [Online]. Available: http://www.itrs.net/ 